Indexed by

\title{
VIBRATION REDUCTION OF CONTINUOUS MOVING LOADS ON A NONLINEAR SIMPLE BEAM RESTING ON AN ELASTIC FOUNDATION
}

Crossref

\section{Yi-Ren Wang}

Tamkang University,

Department of Aerospace

Engineering, New Taipei City,

Taiwan

\section{Chien-Chun Hun}

Tamkang University,

Department of Aerospace

Engineering, New Taipei City,

Taiwan

\section{Hsin Huang}

Tamkang University,

Department of Aerospace

Engineering, New Taipei City, Taiwan

KOBSON

Key words: internal resonance, fixed points plot, vibration, dynamic vibration absorber doi:10.5937/jaes0-30916

Cite article:

Wang Y., Hun C., Huang H. (2022) VIBRATION REDUCTION OF CONTINUOUS MOVING LOADS ON A NONLINEAR SIMPLE BEAM RESTING ON AN ELASTIC FOUNDATION, Journal of Applied Engineering Science, 20(1), 53 - 62, DOI:10.5937/ jaes0-30916

Online aceess of full paper is available at: www.engineeringscience.rs/browse-issues 


\title{
VIBRATION REDUCTION OF CONTINUOUS MOVING LOADS ON A NONLINEAR SIMPLE BEAM RESTING ON AN ELASTIC FOUNDATION
}

\author{
Yi-Ren Wang*, Chien-Chun Hung, Hsin Huang \\ Tamkang University, Department of Aerospace Engineering, New Taipei City, Taiwan
}

This technical note investigates a hinged-hinged nonlinear Euler-Bernoulli beam resting on an elastic foundation subjects to moving loads. The method of multiple scales (MOMS) is employed to analyze this nonlinear beam model. The fixed points plots are made to identify the system's internal resonance. The frequency ratio plot is proposed to predict the system internal resonance conditions. This study improved the author's earlier work for a wider range of prediction on internal resonance conditions. The continuous concentrated moving loads are applied to this nonlinear beam model. The dynamic vibration absorber (DVA) is attached on the beam to reduce vibration and prevent internal resonance. The mass, spring constant and location of the DVA are studied to obtain the best damping effect on the nonlinear beam with moving loads. The results are verified by numerical results and ANSYS simulations.

Key words: internal resonance, fixed points plot, vibration, dynamic vibration absorber

\section{INTRODUCTION}

The vibrations of mechanical elements have always been a concern for researchers and engineers. Mundrey [1] demonstrated the examples of beams placing on elastic foundations which have been widely applied to civil, mechanical, and aerospace engineering. Alam Uzzal [2] considered a linear Euler-Bernoulli beam supported by two-parameter Pasternak foundation subjected to a single moving load. They investigated the deflection and bending moment of the beam for different moving load velocities. Sudheesh Kumar [3] gave an analytical formula for free response of a simple beam under lightly and heavily damped conditions. A single moving load was also considered. Effect of damping at resonant and cancellation of moving speeds is predicted. To reduce the impact of beam vibration, the vibration reduction has usually been accomplished by using the tuned mass damper (TMD) or the dynamic vibration absorber (DVA). Wang and Kuo [4] discussed a hinged-free nonlinear Euler-Bernoulli beam resting on a nonlinear elastic foundation, and found that placing a dynamic vibration absorber (DVA) with appropriate mass could prevent internal resonance and suppress vibrations in the beam. Wang and Lu [5] reported that, in a system with a hinged-hinged nonlinear beam resting on a nonlinear elastic foundation, 1:3 (internal resonance (I.R.)) occurs within the 1st and 3rd modes when the ratio of the elastic modulus of the foundation to that of the beam is . Due to the complicated and limited of the analytical methodology, they just studied the I.R. for the 1st and 3rd modes. Other modes' I.R. conditions were not considered. Samani and Pellicano [6] considered a simple beam with a DVA and sought the optimal DVA location for vibration reduction with concentrated moving loads. They found that a nonlinear DVA has better damping effects than does a linear DVA. However, an I.R. condition needs to be analyzed in the non- linear case. Wang et al. [7] found that when the multiple of mass and the spring constant ( $\mathrm{mDk}$ ) of the TMD shock absorber is a certain value, the best vibration reduction effect can be obtained, and this property still exists when the location of the TMD (ID) is adjusted. Wang and Wei [8] proposed a fluid-convey tube model approximated by a nonlinear beam model resting on an elastic foundation. The different combinations of the damping ring parameters on the beam were studied. The results showed that the instead of TMD's mass and spring \& damping coefficients, the location of the TMD also played an important role in system damping effect. In the present work, the authors' nonlinear beam model [5] is considered. Their analytical result for internal resonance conditions is verified and improved by a simple numerical method. A wider range of I.R. conditions are considered. The continuous concentrated moving loads model is adopted from [6] and added to this nonlinear beam model. The DVA is attached on the beam to reduce vibration and prevent internal resonance. The mass, spring constant and location of the DVA are studied to obtain the best damping effect on the nonlinear beam with moving loads. The method of multiple scales (MOMS) $[9,10]$ is applied to study this nonlinear system in frequency domain. The results are verified by numerical results and ANSYS simulations.

\section{THEORETICAL MODEL}

This research considers a straight beam and assumes that each cross-section of the beam is a plane that follows stress-strain law. Using Newton's 2nd Law, Euler's angle transformation, and Taylor series expansion, this study is able to obtain the basic equations of motion for the nonlinear beam. It is noted that any rotations in the beam are excluded; i.e., limiting it to planar motions. According to the nonlinear 2-D Euler-Bernoulli beam theory 
$[5,10]$, the equation of motion for the $2 \mathrm{D}$ beam (see Fig. 1 ) is shown as:

$$
m \ddot{\ddot{W}}+E I_{A} \bar{W}^{i v}+\bar{\mu} \dot{\bar{W}}+\left[k \bar{W}+\beta \bar{W}^{3}\right]=\frac{E A}{2 \bar{I}} \bar{W}^{\prime \prime} \int_{0}^{T} \bar{W}^{2} d \bar{x}+\bar{F}(\bar{x}, \bar{t})
$$

where $\bar{\mu} \bar{W}$ is the structural damping, $\left[k \bar{W}+\beta \bar{W}^{3}\right]$ are the linear and nonlinear spring term for the elastic foundation, respectively. $\bar{F}=\bar{F}(\bar{x}, \bar{t})$ represents the external load on the beam.

The boundary conditions for the beam are:

$\bar{W}(0, \bar{t})=0, \bar{W}(\bar{I}, \bar{t})=0, \bar{W}^{\prime \prime}(0, \bar{t})=0, \bar{W}^{\prime \prime}(\bar{I}, \bar{t})=0$

The dimensionless equation of motion is expressed as:

$W^{* *}+W^{i v}+\bar{\omega}^{2} W+\mu W^{*}+K W^{3}=\frac{1}{2} \hat{A}\left[\int_{0}^{1} W^{\prime 2} d x\right] W^{\prime \prime}+F(x, T)$

The dimensionless boundary conditions are:

$W(0, \tau)=0 \cdot W(1, \tau)=0 \cdot W^{\prime \prime}(0, T)=0$

where $w=\frac{\bar{W}}{\bar{l}} T=\frac{\bar{t}}{\bar{l}^{2}} \sqrt{\frac{E I_{A}}{m}} \quad x=\frac{\bar{x}}{\bar{l}} \quad l=\frac{\bar{l}}{\bar{l}}=1 \mu=\frac{\bar{\mu} \bar{l}^{2}}{\left(m E l_{A}\right)^{1 / 2}} \hat{A}=\frac{E A}{E I_{A}}$

$\bar{\omega}^{2}=\frac{k \bar{l}^{-4}}{E I_{A}} K=\frac{\beta k \bar{l}^{4}}{E I_{A}}$

In order to simplify the expression of the symbols, this study still uses ( )' and ( () to represent $\mathrm{d} / \mathrm{dx}$ and $\mathrm{d} / \mathrm{d} \mathrm{T}$, respectively in dimensionless equations. The DVA can be treated as a concentrated load on the beam, which can beexpressedas:

\section{$\bar{f}_{s}\left[\bar{W}(\bar{x}, t)-\bar{W}_{D}\right] \delta\left(\bar{x}-\bar{I}_{D}\right)$}

By using the Newton's law, the beam equation with DVA is obtained and in dimensionless form as:

$W^{* *}+W^{i v}+\bar{\omega}^{2} W+\mu W^{*}+K W^{3}+f_{s}\left[W(x, T)-W_{D}\right] \delta\left[x-I_{D}\right]=$

$=\frac{1}{2} \widehat{A}\left[\int_{0}^{1} W^{\prime 2} d x\right] W^{\prime \prime}+F(x, r)$

It is noted that the DVA can be derived by Newton's law and is expressed as:

$m_{D} W_{D}^{* *}(\tau)-f_{s}\left[W(x, T)-W_{D}(\tau)\right]=0$

where $m_{D}$ is the dimensionless mass of the DVA, fs represents the dimensionless spring constant of the DVA, and is defined as

$f_{s}=\frac{\bar{f}_{s}}{m \bar{l} \bar{\omega}^{2}}$

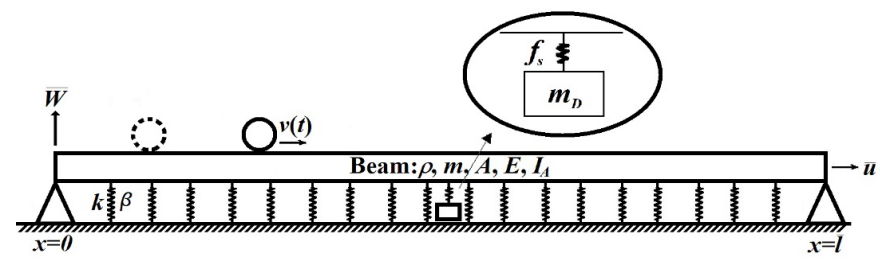

Figure 1: Schematic of the beam model with DVA and moving loads

\section{FORMULATION OF THE MOVING LOAD}

From Ref. [6] the moving load function is written as:

$F(x, t)=\sum_{i=-\infty}^{\infty} F_{0} \delta[x-(v t-i \Delta)] S(i, t), x \in(0, L), t>0$

where

$S(i, t)=H\left[\left(t-\frac{i \Delta}{v}\right)\left(\frac{t}{v}-\left(t-\frac{i \Delta}{v}\right)\right)\right]$

$H(t)=\left\{\begin{array}{l}0, t<0 \\ 1, T>0\end{array}, \int_{-\infty}^{\infty} \delta(t) d t=1,\left\{\begin{array}{c}\delta(t)=0 \text { for } t \neq 0 \\ \delta(0) \text { indefinite }\end{array}\right.\right.$

is the space between the two moving loads, $\delta[x-(v t-i \Delta)]$ represents the location of the $\mathrm{i}^{\text {th }}$ load, $\mathrm{S}(\mathrm{i}, \mathrm{t})$ is a factor to judge if the moving load is located on the beam or not. $\delta(\mathrm{t})$ and $\mathrm{H}(\mathrm{t})$ are the Dirac and Heaviside function, respectively. In this study, the discrete form of the moving load can be obtained by applying the orthogonal property on Eq. (7) and is expressed as:

$\bar{F}_{p}(t)=\int_{0}^{L} \phi_{p}(x) F(x, t) d x=\int_{0}^{L} \phi_{p}(x) \sum_{i=-\infty}^{\infty} F_{0} \delta[x-(v t-i \Delta)] S(i, t) d x=$

$\left.F_{0} S(i, t) \phi_{p}\right|_{x=v t-i \Delta}$

In which $\phi_{p}(x)$ represents the mode shape of the beam and will be shown later in Section 4.1.

The Fourier series is applied and the $\bar{F}_{p}$ is further written as:

$\bar{F}_{p}(t)=\sum_{n=-\infty}^{+\infty} c_{p n} e^{i n \Omega_{p} t} \Rightarrow\left\{\begin{array}{c}c_{p 0}=a_{p 0} \\ c_{p n}=\frac{a_{p n}-i b_{p n}}{2}, c_{-p n}=\frac{a_{p n}+i b_{p n}}{2}\end{array}\right.$

where

$a_{p 0}=\frac{2}{T} \sum_{i=-N L}^{0} \int_{0}^{T} F_{0} S(i, t) \phi_{p}(v t-i \Delta) d t, p=1,2 \ldots$

$a_{p 0}=\frac{2}{T} \sum_{i=-N L}^{0} \int_{0}^{T} F_{0} S(i, t) \phi_{p}(v t-i \Delta) \cos \left(\frac{2 n \pi t}{T}\right) d t, p=1,2 \ldots, n=1,2 \ldots$

$b_{p n}=\frac{2}{T} \sum_{i=-N L}^{0} \int_{0}^{T} F_{0} S(i, t) \phi_{p}(v t-i \Delta) \sin \left(\frac{2 n \pi t}{T}\right) d t, p=1,2 \ldots, n=1,2 \ldots$

and $T=\frac{\Delta}{v}, N L=$ integer part of $L / \Delta$.

The moving frequency of the load is:

$\Omega_{p}=\frac{p \pi v}{L}, p=1,2, \ldots$

\section{I.R. ANALYSIS OF THE BEAM SYSTEM WITH NO DVA}

\section{The frequency ratio plot}

The method of multiple scales (MOMS) is applied to analyze this nonlinear problem in frequency domain. Suppose $T_{0}$ is the fast-time scale term, $T_{1}, T_{2}, \ldots$ are the slowtime scale terms, and let $\mathrm{T}_{0}=\mathrm{T}, \mathrm{T}_{1}=\varepsilon^{2} \mathrm{~T}, .$. the displacement of the beam is expressed as: 
$W(x, T, \varepsilon)=\varepsilon W_{0}\left(x, T_{0}, T_{1} \ldots\right)+\varepsilon^{3} W_{1}\left(x, T_{0}, T_{1} \ldots\right)$

Substituting Eq. (16) in to Eq. (5), we can get the equation of the $\varepsilon^{1}$ order as:

$\frac{\partial^{2} W_{0}}{\partial T_{0}^{2}}+\frac{\partial^{4} W_{0}}{\partial x^{4}}+\bar{\omega}^{2} W_{0}=0$

The equation of the $\varepsilon^{3}$ order is:

$$
\begin{aligned}
& \frac{\partial^{2} W_{1}}{\partial T_{0}^{2}}+\frac{\partial^{4} W_{1}}{\partial x^{4}}+\bar{\omega}^{2} W_{1}=\frac{\partial^{2} W_{0}}{\partial x^{2}} \widehat{A}\left[\frac{1}{2} \int_{0}^{1}\left(\frac{\partial W_{0}}{\partial x}\right)^{2} d x\right]+F- \\
& -f_{2}\left(W_{0}-W_{D}\right) \delta\left(x-I_{D}\right)-2 \frac{\partial^{2} W_{0}}{\partial T_{0} \partial T_{1}}-\mu \frac{\partial W_{0}}{T_{0}}-K W_{0}^{3}
\end{aligned}
$$

The equation of the $\varepsilon^{3}$ order with no DVA is:

$\frac{\partial^{2} W_{1}}{\partial T_{0}^{2}}+\frac{\partial^{4} W_{1}}{\partial x^{4}}+\bar{\omega}^{2} W_{1}=\frac{\partial^{2} W_{0}}{\partial x^{2}} \hat{A}\left[\frac{1}{2} \int_{0}^{1}\left(\frac{\partial W_{0}}{\partial x}\right)^{2} d x\right]+F-2 \frac{\partial^{2} W_{0}}{\partial T_{0} \partial T_{1}}-\mu \frac{\partial W_{0}}{T_{0}}-K W_{0}^{3}$

The boundary conditions for the order of $\varepsilon^{1}$ and $\varepsilon^{3}$ are:

$\varepsilon^{1}: W_{0}(0, r)=0 \cdot W_{0}(1, r)=0 \cdot W_{0}^{\prime \prime}(0, r)=0 \cdot W_{0}^{\prime \prime}(1, \tau)=0$

$\varepsilon^{3}: W_{1}(0, \tau)=0 \cdot W_{1}(1, \tau)=0 \cdot W_{1}^{\prime \prime}(0, r)=0 \cdot W_{1}^{\prime \prime}(1, \tau)=0$

The mode shape for this hinged-hinged beam can also be found as:

$$
\phi_{n}=\sin \gamma_{n} x, n=1,2,3 \ldots \text {, }
$$

where $\phi_{n}$ is the beam's mode shape of the $n^{\text {th }}$ mode, and $Y_{n}$ denotes the eigenvalue of the $n^{\text {th }}$ mode. The I.R. condition can be justified by finding the relationship between modes' frequency ratios and the elastic foundation spring constants. The transverse displacement of the beam is defined as:

$$
W_{0}=\sum_{n=1}^{\infty} \xi_{0 n}(T) \phi_{n}(x), W_{1}=\sum_{n=1}^{\infty} \xi_{1 n}(T) \phi_{n}(x)
$$

Substituting Eq. (22) into Eqs. (17) and (19) to obtain:

Order of $\varepsilon^{1}$

$\sum_{n-1}^{\infty} \phi_{n} \ddot{\xi}_{0 n}+\sum_{n=1}^{\infty}\left(\phi_{n}^{i v}+\bar{\omega}^{2} \phi_{n}\right) \xi_{0 n}=0$

Order of $\varepsilon^{3}$

$\sum_{n=1}^{\infty} \phi_{n} \ddot{\xi}_{1 n}+\sum_{n=1}^{\infty}\left(\phi_{n}^{i v}+\bar{\omega}^{2} \phi_{n}\right) \xi_{1 n}=\sum_{n=1}^{\infty} \ddot{\phi}_{n} \xi_{0 n} \hat{A}\left[\frac{1}{2} \int_{0}^{1}\left(\sum_{n=1}^{\infty} \dot{\phi}_{n} \xi_{0 n}\right)^{2} d x\right]+$

$+F-2 \frac{\partial^{2}}{\partial T_{0} \partial T_{1}} \sum_{n=1}^{\infty} \phi_{n} \xi_{0 n}-\mu \sum_{n=1}^{\infty} \phi_{n} \dot{\xi}_{0 n}-K\left(\sum_{n=1}^{\infty} \phi_{n} \xi_{0 n}\right)$

By using orthogonal property of the mode shapes, the following dynamic equations are obtained:

$\ddot{\xi}_{0 m}+\left(\gamma_{m}^{4}+\bar{\omega}^{2}\right) \xi_{0 m}=0$

$\ddot{\xi}_{1 m}+\left(\gamma_{m}^{4}+\bar{\omega}^{2}\right) \xi_{1 m}=-\frac{1}{2} \gamma_{m}^{2} \xi_{0 m} \hat{A}\left[\int_{0}^{1}\left(\sum_{p=1}^{\infty} \gamma_{p}^{2} \xi_{0 p}^{2} \cos ^{2} \gamma_{p} x\right) d x\right]+\frac{\int_{0}^{1} \phi_{m} d x}{\int_{0}^{1} \phi_{m}^{2} d x} F-$

$-2 \frac{\partial^{2}}{\partial T_{0} \partial T_{1}} \xi_{0 m}-\mu \dot{\xi}_{0 m}-\frac{K}{\int_{0}^{1} \phi_{m}^{2} d x} \int_{0}^{1} \phi_{m} \phi_{n} \phi_{j} \phi_{k} d x \sum_{n, j, k=1}^{\infty} \xi_{0 n} \xi_{0 j} \xi_{0 k}$
The relationship between modal frequency and the foundation spring constant is now expressed as:

$\omega_{m}=\left(\gamma_{m}^{4}+\bar{\omega}^{2}\right)^{\frac{1}{2}}, m=1,2,3 .$.

The I.R. condition can be observed by the plot of different modal frequency ratios with various foundation spring constants $\left(\bar{\omega}^{2}\right)$ as shown in Fig.2. It is noted that due to the lower vibration amplitudes in higher modes (the 4th mode), the energy transferring is not easy to happen between much higher modes and the lower modes. It is also noted that in the case studied and from Eq. (26), the terms of $\xi$ have only orders of 1 and 3 . This implies that only the 1:3 I.R. can be triggered in this system. The frequency ratio of $1: 2$ is not necessary to be considered. Therefore, only the $1^{\text {st }} \& 2^{\text {nd }}$ modes and the $1^{\text {st }} \& 3^{\text {rd }}$ modes with the frequency ratio of $1: 3$ are considered in this study. This study thus provides a wider range of possibilities for I.R. conditions than the methodology proposed by Wang and Lu [5].

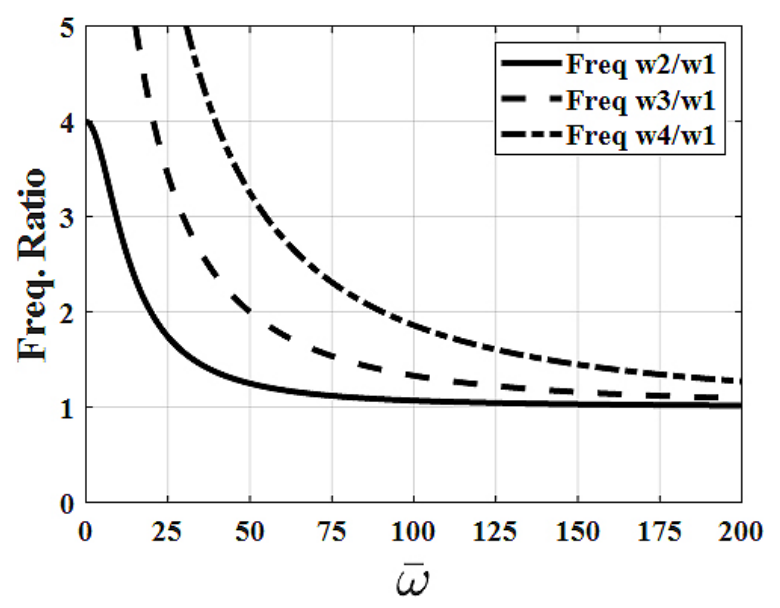

Figure 2: Plot of Freq. Ratio and $\varpi$

\section{Frequency response}

The frequency response of the nonlinear beam system can be got by the fixed points plots. This study gives an example of the 1st and the 3rd modes to demonstrate the frequency response. The general solution of the displacement is assumed as

$\xi_{0 m}(T)=B_{m}\left(T_{1}\right) e^{-i \zeta_{m}} e^{i \omega_{m} T_{0}}+\bar{B}_{m}\left(T_{1}\right) e^{i \zeta_{m}} e^{-i \omega_{m} T_{0}}$

and substituted into Eq. (26) to obtain the following equation:

$\ddot{\xi}_{1 m}+\omega_{m}^{2} \xi_{1 m}=-\frac{1}{2} V_{m}^{2} \hat{A}\left(B_{m}\left(T_{1}\right) e^{-i \zeta_{p}} e^{i \omega_{p} T_{0}}+B_{m}\left(T_{1}\right) e^{i \zeta_{m}} e^{-i \omega_{m} T_{0}}\right)$

$\left(\sum_{p=1}^{\infty} \int_{0}^{1} V_{p}^{2}\left(B_{p}\left(T_{1}\right) e^{-i \zeta_{p}} e^{i \omega_{p} T_{0}}+\bar{B}_{p}\left(T_{1}\right) e^{i \zeta_{p}} e^{-i \omega_{p} T_{0}}\right)^{2} \cos ^{2} \gamma_{p} x\right)-$

$-2\left[i \omega_{m} B_{m}^{\prime}\left(T_{1}\right) e^{-i \zeta_{m}} e^{i \omega_{m} T_{0}}-i \omega_{m} \bar{B}_{m}^{\prime}\left(T_{1}\right) e^{i \zeta_{m}} e^{-i \omega_{m} T_{0}}+\omega_{m} S_{m}^{\prime} \xi_{0 m}\right]-$

$-\mu\left(i \omega_{m} B_{m}\left(T_{1}\right) e^{-i \zeta_{m}} e^{i \omega_{m} T_{0}}-i \omega_{m} \bar{B}_{m}\left(T_{1}\right) e^{i \zeta_{m}} e^{-i \omega_{n} T_{0}}\right)-$

$-\frac{K}{\int_{0}^{1} \sin ^{2} \gamma_{m} x d x}\left[\int_{0}^{1}\left(\sum_{n=1}^{\infty}\left(B_{n}\left(T_{1}\right) e^{-i S_{n}} e^{i \omega_{n} T_{0}}+\bar{B}_{n}\left(T_{1}\right) e^{i S_{n}} e^{-i \omega_{n} T_{0}}\right) \phi_{n}\right)\right.$ 


$$
\left(\sum_{j=1}^{\infty}\left(B_{j}\left(T_{1}\right) e^{-i \zeta_{j}} e^{i \omega_{j} T_{0}}+\bar{B}_{j}\left(T_{1}\right) e^{i \zeta_{j}} e^{-i \omega_{j} T_{0}}\right) \phi_{j}\right)
$$

$\left.\left(\sum_{k=1}^{\infty}\left(B_{k}\left(T_{1}\right) e^{-i \zeta_{k}} e^{i \omega_{k} T_{0}}+\bar{B}_{k}\left(T_{1}\right) e^{i \zeta_{k}} e^{-i \omega_{k} T_{0}}\right) \phi_{k}\right) \phi_{m} d x\right]+F \frac{\int_{0}^{1} \sin \gamma_{m} x d x}{\int_{0}^{1} \sin ^{2} \gamma_{m} x d x}$

The forcing function is assumed to be:

$$
F=f_{m} e^{i \Omega \tau}=f_{m} e^{i\left(\omega_{m}+\varepsilon^{2} \sigma\right) T_{0}}=f_{m}\left(e^{i \varepsilon^{2} \sigma T_{0}} e^{i \omega_{m} T_{0}}\right)=f_{m} e^{i \sigma T_{1}} e^{i \omega_{m} T_{0}}
$$

In which $\sigma$ is the tuned frequency. It is noted that $\sigma=0$ represents the $\Omega$ equals the natural frequency. For the $1^{\text {st }}$ mode $(m=1)$, the secular terms of harmonics of $\omega_{1}$ and $\omega_{3}-2 \omega_{1}$ are selected from the right hand side of Eq. (28). For the $3^{\text {rd }}$ mode $(m=3)$, the secular terms of harmonics of $\omega_{3}$ and $3 \omega_{1}$ are selected from the right hand side of Eq. (28). The solvability conditions are obtained to let the sum of secular terms to be 0 . The fixed points plots are made for the amplitudes of the beam $\left(B_{1}\right.$ and $\left.B_{3}\right)$ with the tuned frequency $(\sigma)$. The I.R. can be analyzed. The mathematical procedure is too complicate and is not detailed here.

\section{Internal resonance analysis}

Figs. 3 and 4 demonstrate the frequency response (fixed points plots) of the $1^{\text {st }}$ and $2^{\text {nd }}$ modes. Fig. 3 and Fig. 4 are the cases when the $1^{\text {st }}$ and the $2^{\text {nd }}$ mode was excited, respectively. Figs. 3(a) and 4(a) are the fixed points plots of the $1^{\text {st }}$ modes. Figs. 3(b) and 4(b) are the fixed points plots of the $2^{\text {nd }}$ mode. Figs. 5 and 6 demonstrate the frequency response (fixed points plots) of the $1^{\text {st }} \&$ $3^{\text {rd }}$ modes. Fig. 5 and Fig. 6 are the cases when the 1 st and the $3^{\text {rd }}$ mode was excited, respectively. Figs. 4 and 6 show that when the higher modes $\left(2^{\text {nd }}\right.$ or $\left.3^{\text {rd }}\right)$ were excited, the lower mode ( $1^{\text {st }}$ mode) still has higher amplitude. This demonstrates the energy transferring from the higher modes to the lower mode(s) and the unexcited mode(s) may have larger amplitudes than the excited mode(s). These results demonstrate the unique characteristics of a nonlinear system. The plot of different modal frequency ratios (Fig.2) plays an important role in analyzing the I.R. conditions.

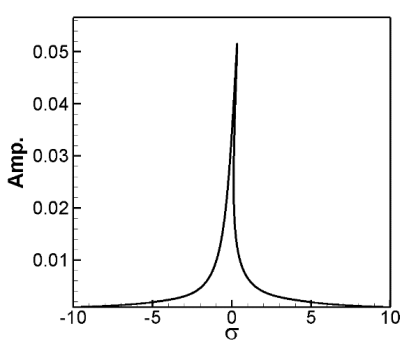

(a)

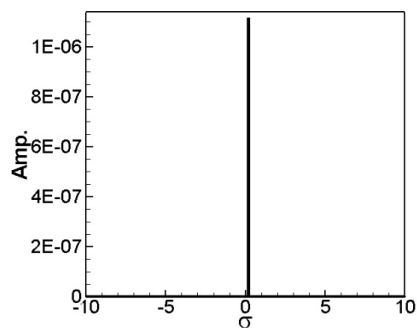

(b)
Figure 3: Fixed points plots with no DVA (1 ${ }^{\text {st }}$ mode was excited) (a) response of the $1^{\text {st }}$ mode, (b) response of the $2^{\text {nd }}$ mode

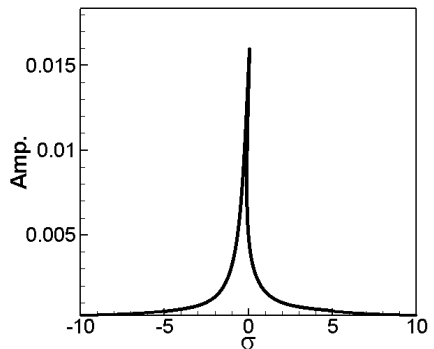

(a)

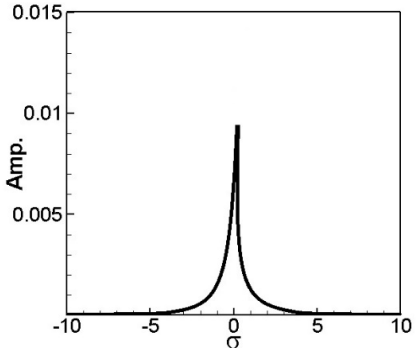

(b)
Figure 4: Fixed points plots with no DVA (2 $2^{\text {nd }}$ mode was excited)(a) response of the $1^{\text {st }}$ mode, (b) response of the $2^{\text {nd }}$ mode

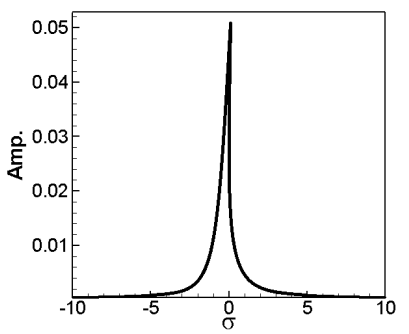

(a)

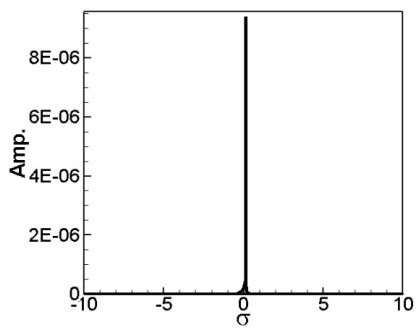

(b)
Figure 5: Fixed points plots with no DVA (1 $1^{\text {st }}$ mode was excited)(a) response of the $1^{\text {st }}$ mode, (b) response of the $3^{\text {rd }}$ mode

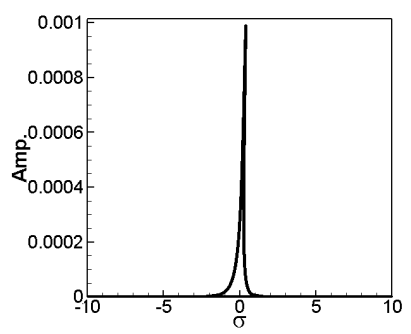

(a)

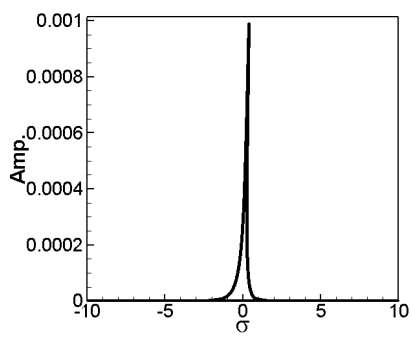

(b)
Figure 6: Fixed points plots with no DVA (3 $3^{\text {rd }}$ mode was excited) (a) response of the $1^{\text {st }}$ mode, (b) response of the $3^{\text {rd }}$ mode

\section{Numerical verification}

The 4th order Runge-Kutta method (RK-4 method) is used to verify the results predicted by Fig. 2 . The small perturbation technique is applied and assuming:

$W=\sum_{n=1}^{\infty}\left(\xi_{n}+\tilde{\xi}_{n}\right) \phi_{n}$

where $\xi_{n}$ is the average term, the $\tilde{\xi}_{n}$ is the perturbed term. Substituting Eq. (30) into Eq. (3) to get the dynamic equation for the RK-4 method. Figs. 7 (a), (b) and (c) are the time responses by using RK-4 to verify the fixed points plots for the 1st mode when the 1st mode was excited, the 1st mode when the 2 nd mode was excited and the 3rd mode when the 3rd mode was excited, respectively. These figures show that the numerical results agree with the semi-analytical predictions from fixed points plots. Again, Fig. 2 shows its valuable in finding 
I.R. conditions.

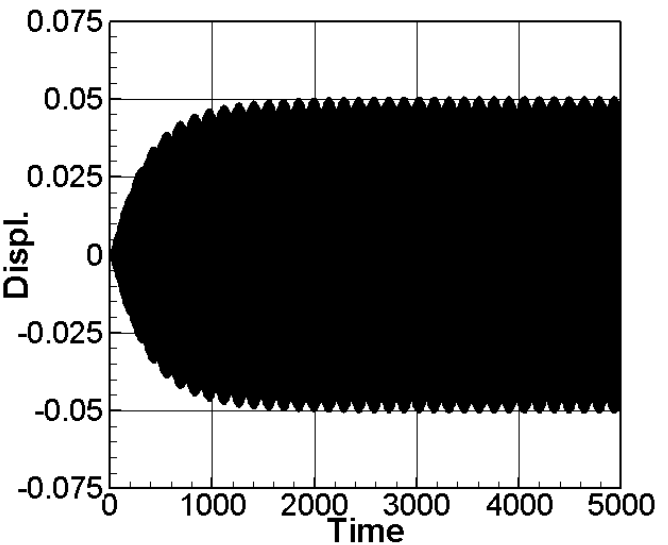

(a)

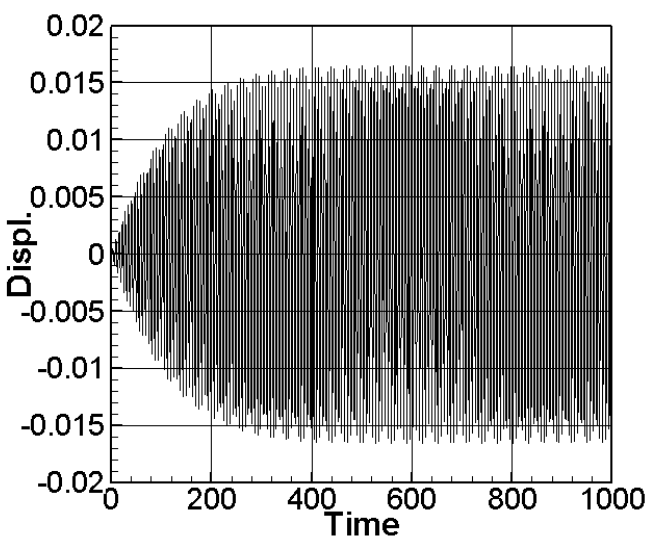

(b)

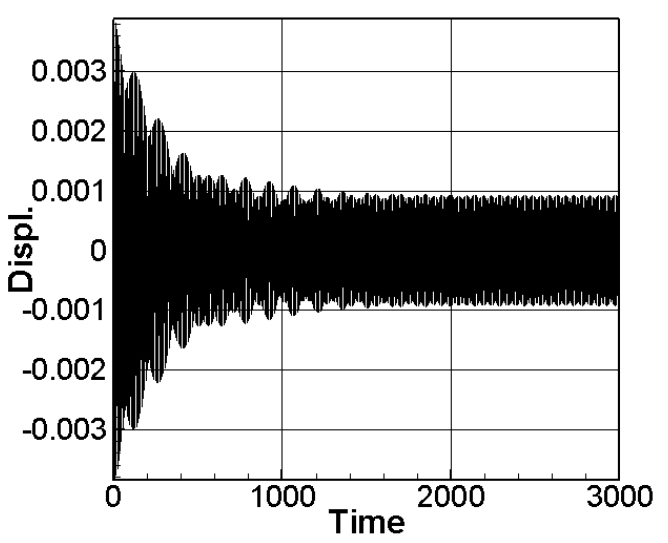

(c)

Figure 7: Numerical verification of fixed points plots (a) to verify Fig. 3(a), (b) to verify Fig. 4(a), (c) to verify Fig. $6(b)$

\section{ANSYS simulation}

This study uses ANSYS to simulate the continuous concentrated moving load on the elastic beam resting on an elastic foundation. The parameters are chosen the same as the data from the work of Samani and Pellicano [6]. The parameters for the steel beam are listed in Table 1. The dimensions are $4000 \mathrm{~mm}, 300 \mathrm{~mm}$ and $300 \mathrm{~mm}$ for the beam's length, width and height, respectively. Solid-
Table 1: Material parameters for the steel beam

\begin{tabular}{|c|c|}
\hline Density $\left(\mathrm{kg} / \mathrm{m}^{3}\right)$ & 7820 \\
\hline Young's Modulus $(\mathrm{Pa})$ & $2.068 \mathrm{E}+11$ \\
\hline Poisson's Ratio & 0.3 \\
\hline
\end{tabular}

Works $^{\mathrm{TM}}$ is used to generate the beam model. The concentrated load is set as $309 \mathrm{~N}$. The concentrated load moving speeds are set from $5 \mathrm{~m} / \mathrm{s} \sim 30 \mathrm{~m} / \mathrm{s}$ and the spaces between loads are $1 \mathrm{~m} \sim 8 \mathrm{~m}$. The ANSYS simulation is performed and the results are collected and made by a 3D plot. For example, Fig. 8 (a) shows the case of no elastic foundation and the moving speed is 25 $\mathrm{m} / \mathrm{s}$, the space between two loads is $6 \mathrm{~m}$. Fig. 8 (b) shows the case of no elastic foundation and the moving speed is $5 \mathrm{~m} / \mathrm{s}$, the space between two loads is $1 \mathrm{~m}$. Fig. 8(a), shows the maximum amplitude is $24.681 \mathrm{~mm}$. It agrees with the result form the prediction from Samani and Pellicano [6], which was $25.27 \mathrm{~mm}$.

Next verification is to study a rather larger loading (e.g. a car) and with an elastic foundation. For this hingedhinged elastic beam, a concentrated moving load of $12250 \mathrm{~N}$ is assumed. The elastic foundation stiffness is set to $1.5925 \mathrm{E}-04 \mathrm{~N} / \mathrm{mm}^{3}$. This value of the foundation stiffness is based on the theoretical prediction for the I.R. condition. The 3D plot of the amplitudes for different combination of moving speed and space is shown in Fig. 9. Fig. 9 shows that when the load moving speed is 26 $\mathrm{m} / \mathrm{s}$ and the space is $8 \mathrm{~m}$, the maximum beam amplitude is $181.79 \mathrm{~mm}$, which agrees with the theoretical prediction of $150.9 \mathrm{~mm}$.

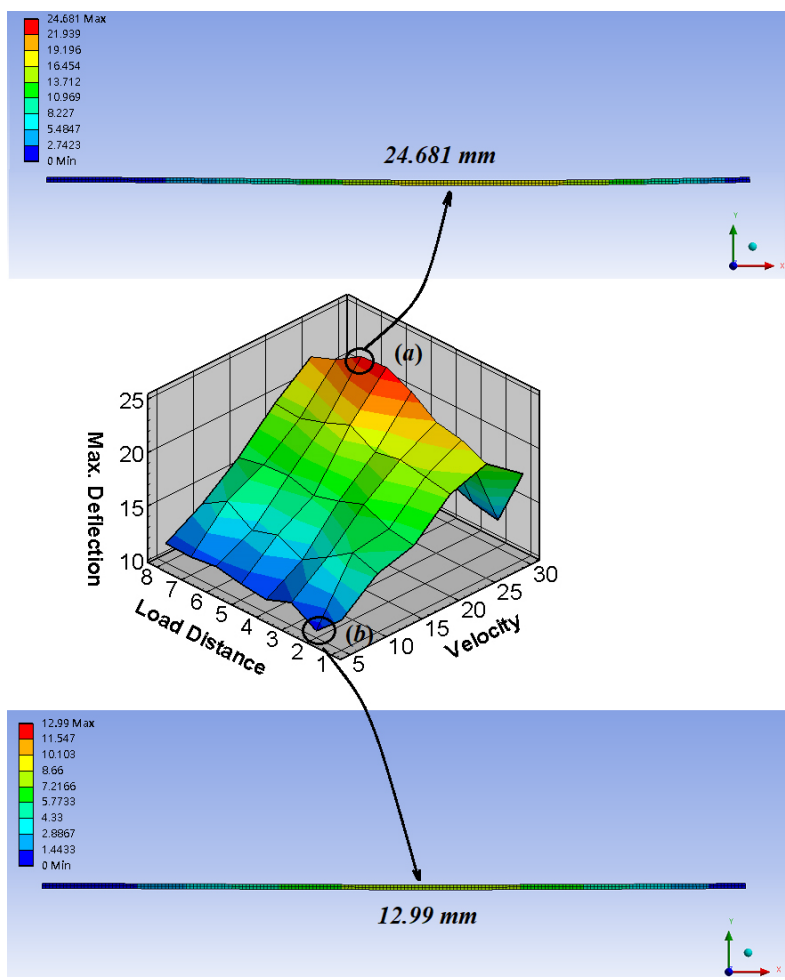

Figure 8: 3D maximum amplitude plot by ANSYS simulation (no elastic foundation) (a) $v=25 \mathrm{~m} / \mathrm{s}, \Delta=6 \mathrm{~m}$, (b) $v=5 \mathrm{~m} / \mathrm{s}, \Delta=1 \mathrm{~m}$ 


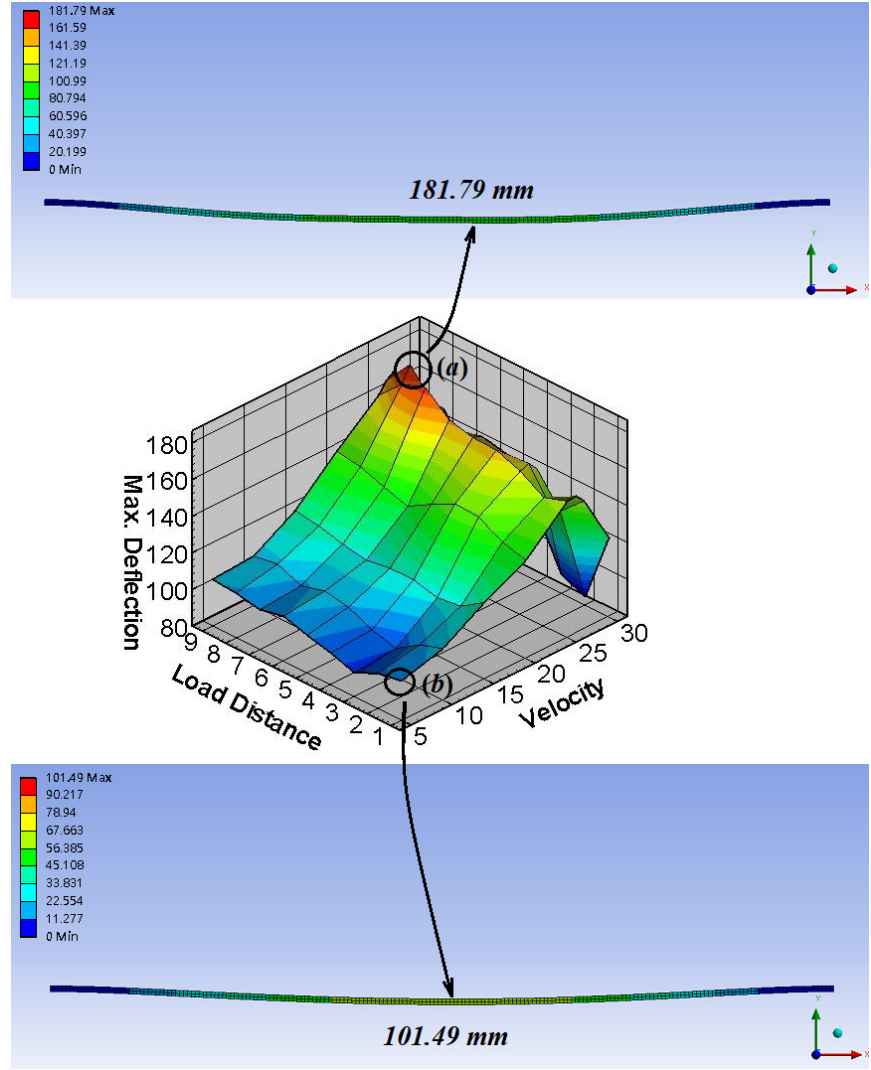

Figure 9: 3D maximum amplitude plot by ANSYS simulation (with elastic foundation) (a) $v=26 \mathrm{~m} / \mathrm{s}, \Delta=8 \mathrm{~m}$, (b) $v=5 \mathrm{~m} / \mathrm{s}, \Delta=1 \mathrm{~m}$

\section{BEAM SYSTEM WITH DVA}

Since I.R. conditions are confirmed. This Section is focusing on the damping effect of the combination of DVA parameters to reduce the beam vibration and to avoid the I.R. of this system to complete a practical engineering application. It is also noted that for the sake of simplicity, only the 1st and the 3rd modes are considered in the beam case with DVA attached.

\section{Theoretical beam model with DVA}

Eq. (6) can be rewritten to include the $1^{\text {st }}$ and the $3^{\text {rd }}$ modes:

$W_{D}^{* *}+\frac{f_{s}}{m_{D}} W_{D}=\frac{f_{s}}{m_{D}} \phi_{1}\left(x_{D}\right)\left(B_{1} e^{-i s_{1}} e^{i \omega_{1} T_{0}}\right)+$

$+\frac{f_{s}}{m_{D}} \phi_{1}\left(x_{D}\right)\left(\bar{B}_{1} e^{i S_{1}} e^{-i \omega_{1} T_{0}}\right)+\frac{f_{s}}{m_{D}} \phi_{3}\left(x_{D}\right)\left(B_{3} e^{-i \zeta_{3}} e^{i \omega_{3} T_{0}}\right)+$

$+\frac{f_{s}}{m_{D}} \phi_{3}\left(x_{D}\right)\left(\bar{B}_{3} e^{i \zeta_{3}} e^{-i \omega_{3} T_{0}}\right)$

The solution of Eq. (31) is assumed as:

$W_{D}=D_{1} e^{-i \zeta_{1}} e^{i \omega_{1} T_{0}}+\bar{D}_{1} e^{i \zeta_{1}} e^{-i \omega_{1} T_{0}}+D_{3} e^{-i \zeta_{3}} e^{i \omega_{3} T_{0}}+\bar{D}_{3} e^{i \zeta_{3}} e^{-i \omega_{3} T_{0}}$

$W_{D}^{*}=i \omega_{1} D_{1} e^{-i \zeta_{1}} e^{i \omega_{1} T_{0}}-i \omega_{1} \bar{D}_{1} e^{i \zeta_{1}} e^{-i \omega_{1} T_{0}}+i \omega_{3} D_{3} e^{-i \zeta_{3}} e^{i \omega_{3} T_{0}}-i \omega_{3} \bar{D}_{3} e^{i \zeta_{3}} e^{-i \omega_{3} T_{0}}$

$W_{D}^{* *}=-\omega_{1}^{2} D_{1} e^{-i \zeta_{1}} e^{i \omega_{1} T_{0}}-\omega_{1}^{2} \bar{D}_{1} e^{i S_{1}} e^{-i \omega_{1} T_{0}}-\omega_{3}^{2} D_{3} e^{-i \zeta_{3}} e^{i \omega_{3} T_{0}}-\omega_{3}^{2} \bar{D}_{3} e^{i \zeta_{3}} e^{-i \omega_{3} T_{0}}$

where

$$
\begin{aligned}
& D_{1}=B_{1} \frac{f_{s} \phi_{1}\left(x_{D}\right)}{f_{s}-m_{D} \omega_{1}^{2}}, \bar{D}_{1}=\bar{B}_{1} \frac{f_{s} \phi_{1}\left(x_{D}\right)}{f_{s}-m_{D} \omega_{3}^{2}} \\
& D_{3}=B_{3} \frac{f_{s} \phi_{3}\left(x_{D}\right)}{f_{s}-m_{D} \omega_{3}^{2}}, \bar{D}_{3}=\bar{B}_{3} \frac{f_{s} \phi_{3}\left(x_{D}\right)}{f_{s}-m_{D} \omega_{3}^{2}} \\
& W_{D}=D_{1} e^{-i s_{1}} e^{i \omega_{1} T_{0}}+\bar{D}_{1} e^{i s_{1}} e^{-i \omega_{1} T_{0}}+D_{3} e^{-i s_{3}} e^{i \omega_{3} T_{0}}+\bar{D}_{3} e^{i \zeta_{3}} e^{-i \omega_{3} T_{0}}
\end{aligned}
$$

Eqs. (33) and (34) can be substituted into Eq. (5) to get the nonlinear beam system with the DVA attached. This study then applies MOMS method for the frequency response analysis. For the sake of simplicity, the procedure of formulating different time scales dynamic equations is not detailed here.

\section{Frequency response analysis}

The forcing function is again assumed as:

$$
F=f_{m} e^{i \Omega \tau}=f_{m} e^{i\left(\omega_{m}+\varepsilon^{2} \sigma\right) T_{0}}=f_{m}\left(e^{i \varepsilon^{2} \sigma T_{0}} e^{i \omega_{m} T_{0}}\right)=f_{m} e^{i \sigma T_{1}} e^{i \omega_{m} T_{0}}
$$

The secular terms are selected and set to 0 to get the solvability conditions as before. Figs. 10 (a) and (b) are the fixed points plots for the 1st mode and the 3rd mode, respectively, when the 1st mode was excited. Figs. 11(a) and (b) are the fixed points plots for the 1st mode and the $3 r d$ mode, respectively, when the 3rd mode was excited. These plots show that no I.R. condition is triggered. There is no energy transferring between the modes when the DVA is applied. Again, RK-4 method is applied and follow the same procedure of Section 4.4; Figs. 12 (a) and (b) provide the verifications of fixed points plots with the numerical results. They agree with each other very well and confirm the results from the fixed points plots.

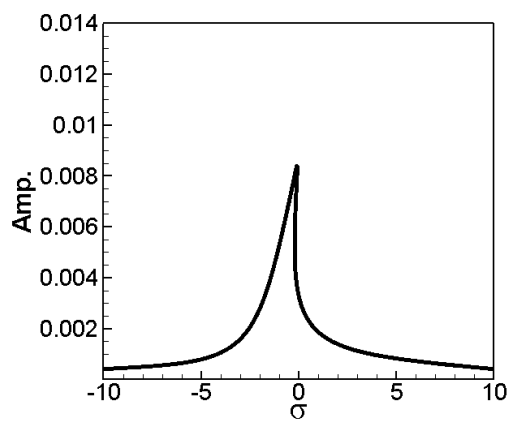

(a)

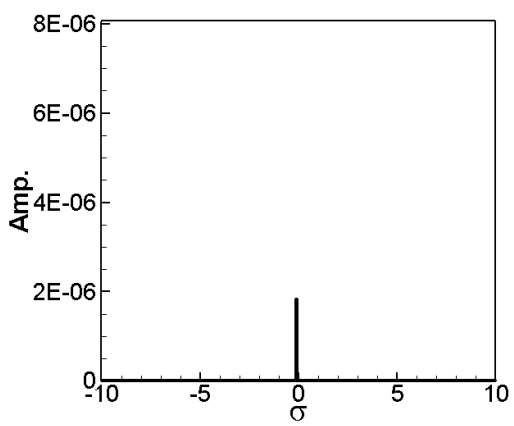

(b)

Figure 10: Fixed points plots with DVA combination $m_{D}$ $=0.1, I_{D}=0.5, f_{s}=9$ (1st mode was excited), (a) response of the $1^{\text {st }}$ mode, (b) response of the $3^{\text {rd }}$ mode 


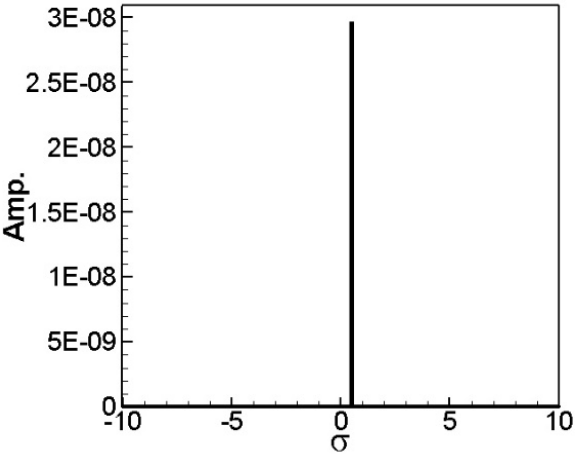

(a)

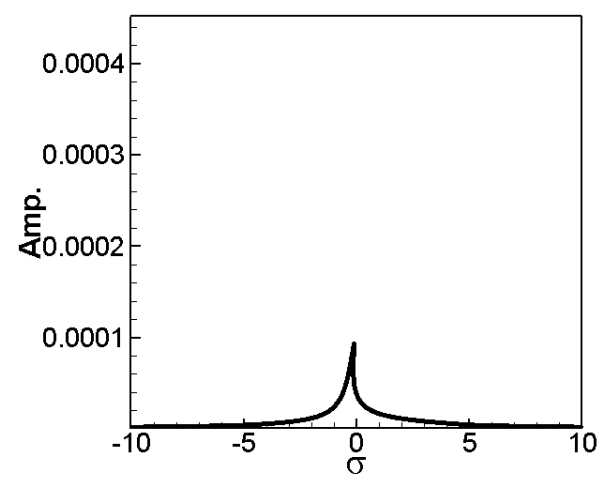

(b)

Figure 11: Fixed points plots with DVA combination $m_{D}$ $=0.1, I_{D}=0.5, f_{s}=9$ ( $3^{\text {rd }}$ mode was excited), (a) response of the $1^{\text {st }}$ mode, (b) response of the $3^{r d}$ mode

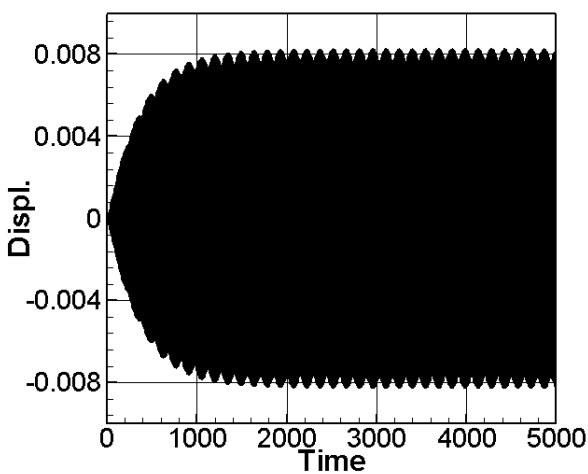

(a)

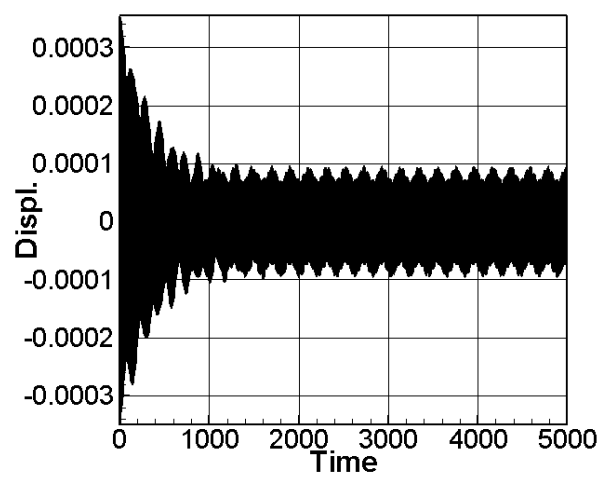

(b)

Figure 12: Numerical verification of fixed points plots (a) to verify Fig. 10(a), (b) to verify Fig. 11(b)

\section{Damping effects of DVA}

This section discusses the vibration reduction effects of DVA according to different mass $\left(m_{D}\right)$, location $\left(I_{D}\right)$, and spring constant $\left(\mathrm{f}_{\mathrm{s}}\right)$. Using the solvability conditions of the excitation to the first mode and the third mode of the system, we can make fixed points plots according to the different combinations of DVA parameters $\left(m_{D}=0.01 \sim 0.1\right.$, $\mathrm{I}_{\mathrm{D}}=0.1 \sim 0.5, \mathrm{f}_{\mathrm{s}}=1,5,9$ ), and extract their maximum amplitudes from each fixed points plot, so we can get the maximum amplitude of each mode when the system is affected by different DVA parameters. Since the amplitude of the third mode (see Fig.5(b), approximates to 9.6E-006), is much smaller than that of the first mode when the first mode was excited, this study does not analyze the vibration reduction of the third mode when the first mode was excited. The amplitude of the first mode (see Fig.11) is much smaller than that of the third mode when the third mode was excited, this study does not analyze the vibration reduction of the first mode when the third mode was excited. The results of vibration reduction are shown in Tables. 2 and 3. Table 2 shows the 1 st mode maximum amplitudes when the $1^{\text {st }}$ mode was excited and for different combinations of $m_{D}, I_{D}$ and $f_{s}$. Table 3 demonstrates the $3^{\text {rd }}$ mode maximum amplitudes when the $3^{\text {rd }}$ mode was excited and for different combinations of $m_{D}, l_{D}$ and fs. Tables 2 and 3 conclude that the combination of $m_{D}$ $=0.1, I_{D}=0.5$ and $f_{s}=9$ provides the best damping effect on this beam vibration system. The corresponding amplitudes for the 1st mode and the $3^{\text {rd }}$ mode are 0.008398 and 0.0000935 , respectively.

The damping effects can be seen by comparing with no DVA system. Figs. 10 and 11 are the fixed points plots with the DVA combination of $m_{D}=0.1, l_{D}=0.5$ and $f_{s}=9$ and for the $1^{\text {st }}$ and $3^{\text {rd }}$ mode, respectively. Figs. 5 and 6 are the plots for no DVA attached. Comparing with Figs. 10 and 5 and Figs. 11 and 6 , the damping effects of the DVA can be obviously demonstrated.

Finally, this study uses ANSYS to simulate the damping effects of the DVA combination with a moving load. The case of maximum amplitude with no DVA attached is considered, which is the moving load speed $v=26 \mathrm{~m} / \mathrm{s}$ and the moving load space $\Delta=8 \mathrm{~m}$. The mass of the DVA is $m_{D}=0.1$ with the length $=400 \mathrm{~mm}$, width $=30 \mathrm{~mm}$, and height $=30 \mathrm{~mm}$. The simulation results are shown in Figs. 13 15. Fig. 13 is the case of DVA combination of $m_{D}=0.1, l_{D}=0.5$ and $f_{s}=9$. The amplitude is $38.042 \mathrm{~mm}$ from ANSYS simulation. The theoretical prediction is shown on the last row in Table 2 (0.008398 (dimensionless displacement)). For a beam of $4000 \mathrm{~mm}$, the theoretical beam displacement is $33.592 \mathrm{~mm}$. The error with the theoretical prediction is $11.7 \%$. Fig. 14 is the case of DVA combination of $m_{D}=0.02, I_{D}=0.25$ and $f_{s}=1$. The amplitude is $46.584 \mathrm{~mm}$ from ANSYS simulation. The theoretical prediction is shown on the first row in Table 2 (0.009746 (dimensionless displacement)). The error with the theoretical prediction is $16.3 \%$. Fig. 15 is the case of DVA combination of $m_{D}=0.06, I_{D}=0.25$ and $f_{s}=5$. The am- 
Table 2: The $1^{\text {st }}$ mode beam amplitude with DVA attached, when the $1^{\text {st }}$ mode is excited

\begin{tabular}{|c|c|c|c|c|}
\hline \multirow{2}{*}{ DVA Mass } & \multirow{2}{*}{ DVA Position } & \multicolumn{3}{|c|}{ Amp. } \\
\cline { 3 - 5 } & & $\mathrm{f}_{\mathrm{s}}=1$ & $\mathrm{f}_{\mathrm{s}}=5$ & $\mathrm{f}_{\mathrm{s}}=9$ \\
\hline 0.02 & \multirow{3}{*}{0.25} & 0.009746 & 0.009327 & 0.008926 \\
0.06 & & 0.009570 & 0.008937 & 0.008883 \\
0.1 & & 0.009275 & 0.008639 & 0.008536 \\
\hline 0.02 & \multirow{3}{*}{0.5} & 0.009624 & 0.008788 & 0.008866 \\
0.06 & 0.008793 & 0.008470 & 0.008574 \\
0.1 & & 0.008490 & 0.008415 & 0.008398 \\
\hline
\end{tabular}

Table 3: The $3^{\text {rd }}$ mode beam amplitude with DVA attached, when the $3^{\text {rd }}$ mode is excited

\begin{tabular}{|c|c|c|c|c|}
\hline \multirow{2}{*}{ DVA Mass } & \multirow{2}{*}{ DVA Position } & \multicolumn{3}{|c|}{ Amp. } \\
\cline { 3 - 5 } & & $\mathrm{f}_{\mathrm{s}}=1$ & $\mathrm{f}_{\mathrm{s}}=5$ & $\mathrm{f}_{\mathrm{s}}=9$ \\
\hline 0.02 & \multirow{3}{*}{0.25} & 0.0001073 & 0.0001062 & 0.0001053 \\
0.06 & & 0.0001069 & 0.0001061 & 0.0001045 \\
0.1 & & 0.0001042 & 0.0000976 & 0.0000968 \\
\hline 0.02 & \multirow{3}{*}{0.5} & 0.0001055 & 0.0001037 & 0.0001021 \\
0.06 & & 0.0001053 & 0.0001032 & 0.0001014 \\
0.1 & & 0.0001050 & 0.0000948 & 0.0000935 \\
\hline
\end{tabular}

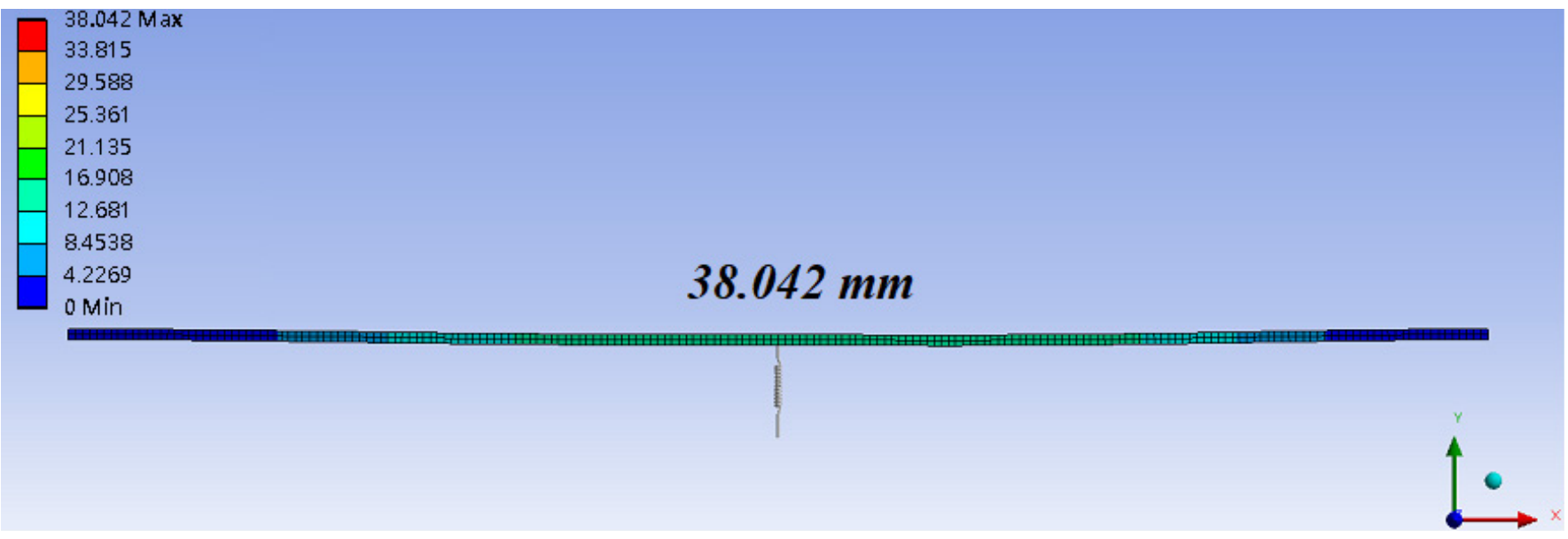

Figure 13: ANSYS simulation with DVA, $m_{D}=0.1, I_{D}=0.5, f_{s}=9$

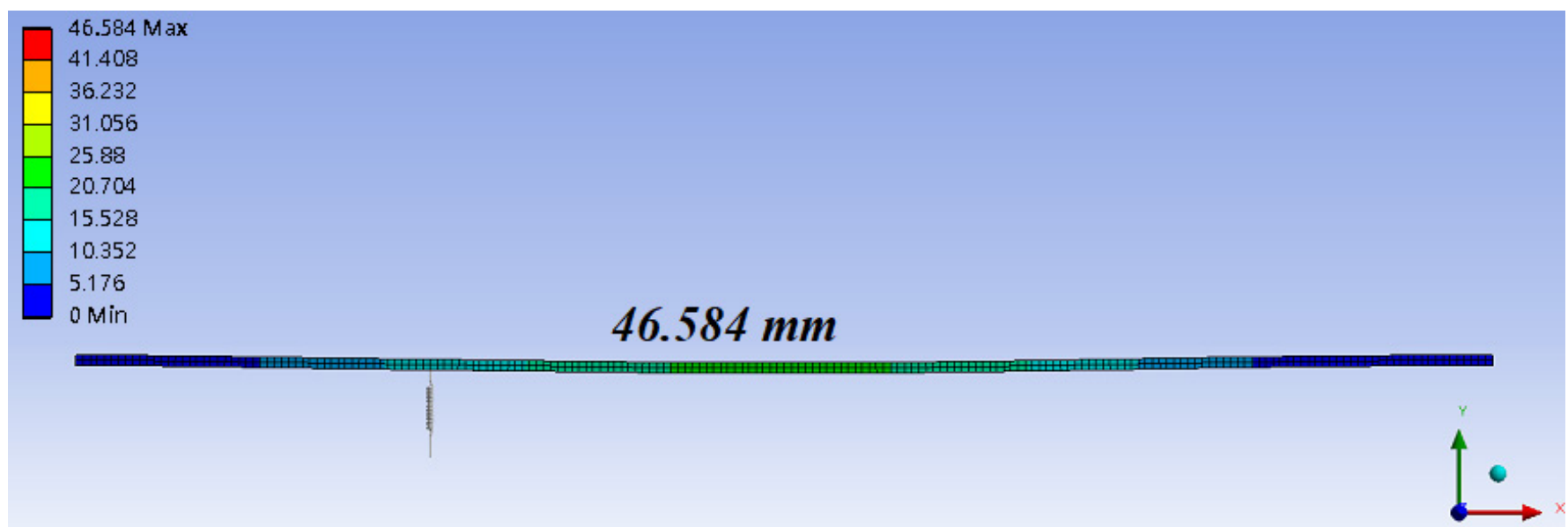

Figure 14: ANSYS simulation with DVA, $m_{D}=0.02, I_{D}=0.25, f_{s}=1$ 


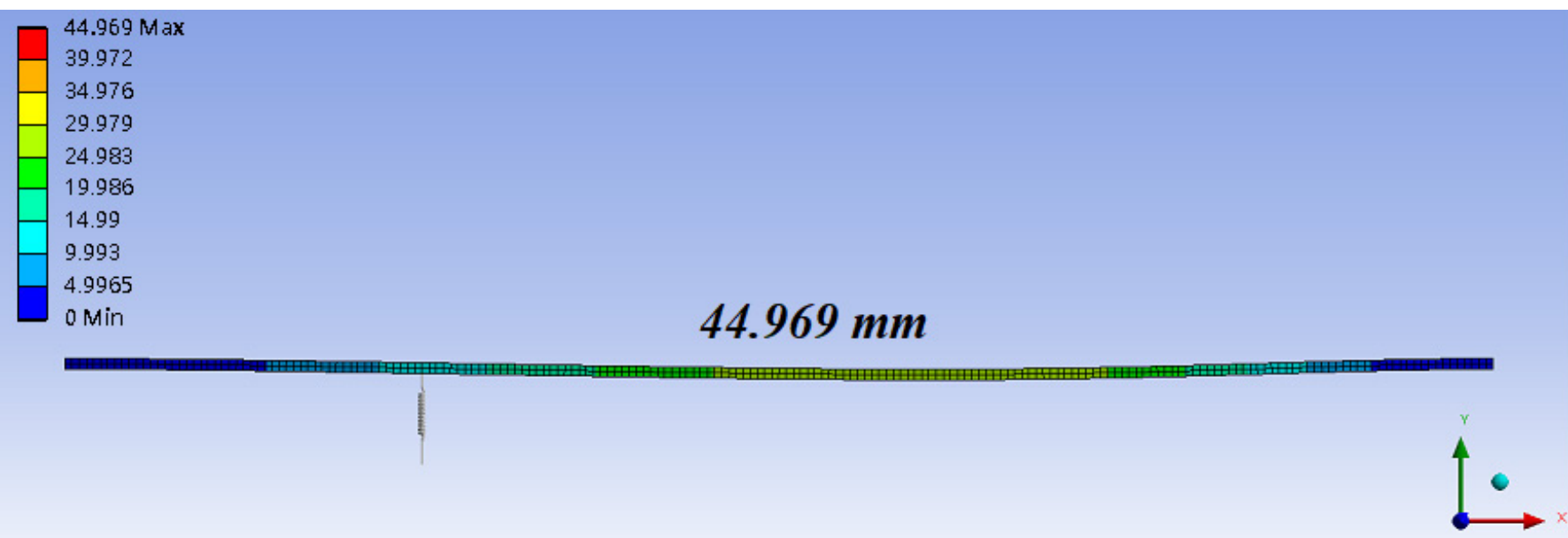

Figure 15: ANSYS simulation with DVA, $m_{D}=0.06, I_{D}=0.25, f_{s}=5$

Table 4: Comparison between theoretical and simulated amplitudes

\begin{tabular}{|c|c|c|c|}
\hline Case studied (Amp.) & $\begin{array}{c}\text { Theoretical prediction } \\
(\mathbf{m m})\end{array}$ & ANSYS Simulation $(\mathbf{m m})$ & Error $(\%)$ \\
\hline $\mathrm{m}_{\mathrm{D}}=0.1, \mathrm{I}_{\mathrm{D}}=0.5, \mathrm{f}_{\mathrm{s}}=9$ & 33.592 & 38.042 & $11.7 \%$ \\
\hline $\mathrm{m}_{\mathrm{D}}=0.02, \mathrm{I}_{\mathrm{D}}=0.25, \mathrm{f}_{\mathrm{s}}=1$ & 38.984 & 46.584 & $16.3 \%$ \\
\hline $\mathrm{m}_{\mathrm{D}}=0.06, \mathrm{I}_{\mathrm{D}}=0.25, \mathrm{f}_{\mathrm{s}}=5$ & 35.748 & 44.969 & $20.5 \%$ \\
\hline
\end{tabular}

plitude is $44.969 \mathrm{~mm}$ from ANSYS simulation. The theoretical prediction is shown on the second row in Table 2 (0.008937 (dimensionless displacement)). The error with the theoretical prediction is $20.5 \%$. An explicit tabular comparison between theoretical and simulated amplitudes is shown on Table 4. Since the theoretical predictions are based on the nonlinear assumption, the energy transferring between lower and higher modes is considered. The ANSYS simulation results are linear, and the beam deformation directly responds to the excited load. In other words, in a linear system, the energy from the external load directly delivers to a single beam mode. However, the errors are almost less than $20 \%$ and the trends are the same as the theoretical results. The nonlinear model proves its application in practical problems.

\section{CONCLUSIONS}

This study improves the author's earlier work (Wang and Lu [5]) for a wider range of prediction on internal resonance conditions. The continuous concentrated moving load is applied on this nonlinear beam system. Instead of complicated methodology and calculation, this study provides an extended numerically graphical plot for different modal frequency ratios and the foundation spring constant, which demonstrates a better way to identify the internal resonance conditions of the nonlinear system. The results show that the $1^{\text {st }} \&$ the $2^{\text {nd }}$ and also the $1^{\text {st }} \&$ the $3^{\text {rd }}$ modes are possible to trigger the I.R., which was not predicted in Wang and Lu's work ([5]). The fixed points plots are made to verify this finding. The moving load with moving speed $\mathrm{v}=26 \mathrm{~m} / \mathrm{s}$ and the space $\Delta=8 \mathrm{~m}$ between the loads causes the resonant condition of the beam system and has the maximum vibration amplitude. The DVA with the combination of $m_{D}=0.1, l_{D}=0.5$ and $f_{s}=9$ provides the best damping effect on this beam system. Both numerical and ANSYS simulations verify this finding.

\section{ACKNOWLEDGEMENT}

This research was supported by the Ministry of Science and Technology of Taiwan, Republic of China (Title of the project: Analysis and verification of space rockets subproject: Vibration reducing on space rockets. Grant number: MOST 109-2224-E-006-004).

\section{REFERENCES}

1. Mundrey, J. S. (2000). Railway Track Engineering. Tata McGraw-Hill, New Delhi.

2. Uzzal, R.U.A., Bhat, R. B. and Ahmed, W. (2012). Dynamic response of a beam subjected to moving load and moving mass supported by Pasternak foundation. Shock and Vibration, vol. 19, 205-220, DOI: https://doi.org/10.3233/SAV-2011-0624

3. Sudheesh Kumar, C.P., Sujatha, C. and Shankar, K. (2015). Vibration of simply supported beams under a single moving load: A detailed study of cancellation phenomenon. International Journal of Mechanical Sciences, vol. 99, 40-47, DOI: https://doi.org/ 10.1016/j.jimecsci.2015.05.001

4. Wang, Y.R. and Kuo, T.H. (2016). Effects of a Dynamic Vibration Absorber on Nonlinear Hingedfree Beam. ASCE Journal of Engineering Mechanics, vol.142, no. 4, 25 pages, DOI: https://doi. org/10.1061/(ASCE)EM.1943-7889.0001039 
5. Wang, Y.R. and Lu, H.C. (2017). Damping performance of dynamic vibration absorber in nonlinear simple beam with 1:3 internal resonance. International Journal of Acoustics and Vibration, vol.22, no.2, 167-185, DOI: 10.20855/ijav.2017.22.2462

6. Samani, F.S. and Pellicano, F. (2012). Vibration reduction of beams under successive traveling loads by means of linear and nonlinear dynamic absorbers. Journal of Sound and Vibration, vol. 331, no. 10, 2272-2290, DOI: https://doi.org/10.1016/j. jsv.2012.01.002

7. Wang, Y.R., Feng, C.K. and Chen, S.Y. (2018). Damping effects of linear and nonlinear tuned mass dampers on nonlinear hinged-hinged beam. Journal of Sound and Vibration, vol. 430, 150-173, DOI: https://doi.org/10.1016/j.jsv.2018.05.033
8. Wang, Y.R. and Wei, Y.H. (2020). Internal resonance analysis of a fluid-conveying tube resting on a nonlinear elastic foundation. Eur. Phys. J. Plus, vol.135, Article number 364, DOI: https://doi.org/10.1140/ epjp/s13360-020-00353-4

9. Nayfeh, A. H. and Mook, D. T. (1995). Ch.4 Forced Oscillations of Systems Having a Single Degree of Freedom. Nonlinear Oscillations. Wiley-Interscience, New York, p.161-257

10. Nayfeh, A.H. and Pai, P.F. (2004). Ch.4 Beams. Linear and Nonlinear Structural Mechanics. Wiley-Interscience Publication, New York, p.171-265. 